\begin{tabular}{|c|l|}
\hline Title & $\begin{array}{l}\text { Continuous alignment of vorticity direction prevents the blow-up of the navier-stokes flow under the no-slip boundary } \\
\text { condition }\end{array}$ \\
\hline Author(s) & Giga, Y oshikazu; Gu, Zhongy ang; Hsu, Pen-yuan \\
\hline Citation & Hokkaido University Preprint Series in Mathematics, 1125, 1-12 \\
\hline Issue Date & 2019-0424 \\
\hline DOI & 10.14943/88214 \\
\hline Doc URL & http:/hdl.handle.net/2115/73710 \\
\hline Type & bulletin (article) \\
\hline File Information & ContinuousA lignmentV orticity.pdf \\
\hline
\end{tabular}

Instructions for use 


\title{
CONTINUOUS ALIGNMENT OF VORTICITY DIRECTION PREVENTS THE BLOW-UP OF THE NAVIER-STOKES FLOW UNDER THE NO-SLIP BOUNDARY CONDITION
}

\author{
YOSHIKAZU GIGA, ZHONGYANG GU, AND PEN-YUAN HSU
}

\begin{abstract}
This paper is concerned with a regularity criterion based on vorticity direction for Navier-Stokes equations in a three-dimensional bounded domain under the no-slip boundary condition. It asserts that if the vorticity direction is uniformly continuous in space uniformly in time, there is no type I blow-up. A similar result has been proved for a half space by Y. Maekawa and the first and the last authors (2014). The result of this paper is its natural but non-trivial extension based on $L^{\infty}$ theory of the Stokes and the Navier-Stokes equations recently developed by K. Abe and the first author.
\end{abstract}

\section{INTRODUCTION}

We are interested in a regularity criterion based on vorticity direction for the three-dimensional Navier-Stokes equations under the no-slip boundary condition. To be specific, let us consider the Navier-Stokes system of the form

$$
\partial_{t} u-\Delta u+\operatorname{div}(u \otimes u)+\nabla p=0, \quad \operatorname{div} u=0 \quad \text { in } \quad U \times(-1,0)
$$

with the no-slip boundary condition

$$
u=0 \quad \text { on } \quad \partial U \times(-1,0) .
$$

Here $U$ is a domain in $\mathbf{R}^{3}$ and $u=u(x, t)=\left(u_{1}(x, t), u_{2}(x, t), u_{3}(x, t)\right), p=$ $p(x, t)$ denotes the velocity field and the pressure field, respectively. We use the standard notion for derivatives; $\partial_{t}=\partial / \partial t, \partial_{j}=\partial / \partial x_{j}, \Delta=\sum_{j=1}^{3} \partial_{j}^{2}$, $\operatorname{div} u=\sum_{j=1}^{3} \partial_{j} u_{j}, u \otimes u=\left(u_{i} u_{j}\right)_{1 \leq i, j \leq 3}$ and $\operatorname{div} F=\sum_{j=1}^{3} \partial_{j} F_{i j}$ for a tensor field $F=\left(F_{i j}\right)_{1 \leq i, j \leq 3}$.

In [15] it has been proved that continuous alignment of vorticity direction prevents the blow-up of a solution of (1.1) when $U=\mathbf{R}^{3}$. Here is a precise statement. Let $\omega=\operatorname{curl} u$ denote the vorticity field.

Key words and phrases. vorticity direction; $L^{\infty}$ theory; Liouville type theorem.

The work of the first author was partly supported by the Japan Society for the Promotion of Science through the grant No. 26220702 (Kiban S), No. 19H00639 (Kiban A), No. 18H05323 (Kaitaku), No. 17H01091 (Kiban A) and No. 16H03948 (Kiban B). 
Theorem $1.1([15])$. Let $(u, p)$ be a spatially bounded mild solution to the Navier-Stokes system (1.1) with $U=\mathbf{R}^{3}$. Assume that the possible blow-up of $u$ at time 0 is type I, i.e.,

$$
\sup _{-1<t<0}(-t)^{1 / 2}\|u(t)\|_{\infty}<\infty .
$$

Let $d$ be a positive number and let $\eta$ be a non-decreasing continuous functions on $[0, \infty)$ satisfying $\eta(0)=0$. Assume that $\eta$ is a modulus of continuity in the $x$ variables for the vorticity direction $\xi=\omega /|\omega|$, in the sense that

$$
|\xi(x, t)-\xi(y, t)| \leq \eta(|x-y|) \quad \text { for } \quad(x, t), \quad(y, t) \in Q_{d},
$$

where $Q_{d}=\{(x, t) \in Q=U \times(-1,0)|| \omega(x, t) \mid>d\}$. Then $u$ is bounded up to $t=0$.

Intuitively speaking, this theorem says that the type I blow-up does not occur unless vorticity direction is wildly changing depending on space. The condition (1.3) is often called a "continuous alignment" condition. This phenomenon is numerically observed for example in [19]. As remarked in [15], this result can be easily extended to the case when $U$ has a boundary under slip-boundary condition but it is non-trivial to extend this result under the no-slip boundary condition (1.2) even if $U$ is a half space $\mathbf{R}_{+}^{3}=\left\{\left(x_{1}, x_{2}, x_{3}\right) \in \mathbf{R}^{3} \mid x_{3}>0\right\}$.

Fortunately, this problem can be overcome by establishing an interesting Liouville type theorem for the two-dimensional Navier-Stokes flow in a half space [14].

Theorem 1.2 ([14]). The statement of Theorem 1.1 is still valid under the boundary condition (1.2) at least when $U=\mathbf{R}_{+}^{3}$.

The goal of this paper is to extend this result when $U$ has a curved boundary. This is highly nontrivial at least technically since we appeal a blow-up argument. As we see later, our argument depends on compactness of rescaled pressure and for this purpose we need $L^{\infty}$-theory for the Stokes and the Navier-Stokes flow which is easy when $U=\mathbf{R}^{3}$ and can be carried out when $U=\mathbf{R}_{+}^{3}$ by explicit formulas [7], [30], [26]. If the boundary has a curved part, to establish $L^{\infty}$-theory is nontrivial. Recent development of $L^{\infty}$ theory (e.g. K. Abe [2]) enables us to prove

Theorem 1.3. The statement of Theorem 1.1 is still valid under the boundary condition (1.2) when $U$ is a $C^{3}$ bounded domain in $\mathbf{R}^{3}$.

Our goal is to prove Theorem 1.3 by a blow-up argument. This kind of a non-blow up criterion based on the vorticity direction was first given in [13] for a finite energy solution for $U=\mathbf{R}^{3}$ with $H^{1}$ initial data and $\eta(\sigma)=A \sigma, A>0$, i.e., $\xi$ is Lipschitz in space uniformly in time. Their method is a kind of energy method. They do not assume type I. There are several extensions of this result, [10], [8], [9]. The reader is referred to a review paper [11] and [15] for further references. We note that under the 
no-slip boundary condition, it often needs an extra assumption. In [9], it is assumed that the boundary integral of the normal derivative of the square of the vorticity is sufficiently small. Even for the half space Theorem 1.2 seems to be the first contribution to the case of no-slip boundary condition under the same assumption to the whole space.

Our strategy is to appeal a blow-up argument based on contradiction argument which was used for the Navier-Stokes equations in [21] and independently by [15], [14]; see also a review paper [29]. It consists of two parts: compactness of blow-up sequence and uniqueness of its limit based on a Liouville type theorem. For the first step, we need an $L^{\infty}$-theory for the Navier-Stokes equations [2], [27] for a domain with curved boundary. To establish a good $L^{\infty}$-theory, we need a control of pressure even for the Stokes equations [5], [6]. To study the nonlinear problem, we also need a control of pressure due to convective terms.

The second step is to use a Liouville type theorem for ancient solutions available in [14]. There are various Liouville type theorems for the NavierStokes equations which are useful for the blow-up argument. For example, the Liouville type theorem for ancient bounded solutions in a whole plane is given in [21] and [15]. The proof in [21] is based on integral estimates while in [15] it was proved by a simple shifting argument. The latter argument was rediscovered by [24], apparently without knowing [15]. For axisymmetric flows, several Liouville type theorems have been established in [21] and [23], [24] to derive non-blow up criteria for such flows. However, we note that there seem to have no Liouville type results for the half plane with the Dirichlet condition for ancient solutions without assuming spatial decay except [14]. The reader is referred to a review paper [29] for blow-up argument and Liouville type theorems for the Navier-Stokes flow as well as regularity criteria.

\section{Blow-UP ARGUMENT}

We shall recall a blow-up argument in [15], [14] to prove Theorem 1.3.

2.1. Construction of a blow-up sequence. Suppose that $u$ blows up at $t=0$, i.e., $\lim _{t \rightarrow 0}\|u(t)\|_{\infty}=\infty$. Then there would exist a sequence $\left\{\left(x_{k}, t_{k}\right)\right\}_{k=1}^{\infty} \subset Q$ with $t_{k+1}>t_{k}$ such that

(i) $|u(x, t)| \leq M_{k}$ for $t \leq t_{k}, x \in U$,

(ii) $M_{k}=\left\|u\left(t_{k}\right)\right\|_{\infty} \rightarrow \infty, t_{k} \uparrow 0$ as $k \rightarrow \infty$,

(iii) $\left|u\left(x_{k}, t_{k}\right)\right| \geq M_{k} / 2$.

We rescale $u, \omega$ with respect to $\left(x_{k}, t_{k}\right)$, i.e.,

$$
\begin{aligned}
& u_{k}(x, t)=\lambda_{k} u\left(x_{k}+\lambda_{k} x, t_{k}+\lambda_{k}^{2} t\right) \\
& \omega_{k}(x, t)=\lambda_{k}^{2} \omega\left(x_{k}+\lambda_{k} x, t_{k}+\lambda_{k}^{2} t\right),-t_{k}-1<\lambda_{k}^{2} t<-t_{k}
\end{aligned}
$$

with $\lambda_{k}=1 / M_{k}$. By the scaling invariant property of the Navier-Stokes equations we see that $u_{k}$ is still a mild solution of (1.1), (1.2) in $U_{k} \times$ 
$\left(-\left(t_{k}+1\right) M_{k}^{2}, 0\right]$ with

$$
U_{k}=\left\{x \in \mathbf{R}^{3} \mid x_{k}+\lambda_{k} x \in U\right\} .
$$

We shall study several compactness of the sequence. One is $L^{\infty} *$-weak compactness of $\left(u_{k}, \omega_{k}\right)$. Another one is $L^{\infty}$ strong compactness near $(0,0)$.

2.2. Weak compactness. By Assumption (i) we have $\left|u_{k}\right| \leq 1$ in $U_{k} \times$ $\left(-\left(t_{k}+1\right) M_{k}^{2}, 0\right]$. Since $u_{k}$ is a mild solution, we apply the following uniqueness and existence theorem to conclude that $\nabla u_{k}$ is also bounded in $U_{k} \times$ $\left(-\left(t_{k}+1\right) M_{k}^{2}+1,0\right]$.

Theorem 2.1 ([2]). Let $U$ be a bounded or exterior domain in $\mathbf{R}^{n}$ with $C^{3}$ boundary. There exists a positive constant a such that for $u_{0} \in C_{0, \sigma}$ there exists $T \geq a /\left\|u_{0}\right\|_{\infty}^{2}$ and a unique mild solutions $u \in C\left([0, T], C_{0, \sigma}\right)$ of the Navier-Stokes system in $U \times(0, T)$ with the zero Dirichlet boundary condition and $\left.u\right|_{t=0}=u_{0}$. The solution $u$ satisfies $t^{1 / 2} \nabla u \in C\left([0, T], L^{\infty}\right)$.

Remark 2.2. This result is based on the $L^{\infty}$-theory of the Stokes semigroup [5], [6] and its estimates involving gradents and the Helmholtz projection [3], [4]. This result is extended when initial data $u_{0}$ is in $L_{\sigma}^{\infty}$ (solenoidal $L^{\infty}$ space) or $u_{0}$ is in $B U C_{\sigma}$ (solenoidal space of bounded uniformly continuous functions vanishing on $\partial U$ ) by [4]. A different proof for $u_{0} \in B U C_{\sigma}$ is given by P. Maremonti [27] which also depends on the linear estimate by [5], [6].

Here $C_{0, \sigma}=C_{0, \sigma}(U)$ denotes $L^{\infty}$-closure of $C_{c, \sigma}^{\infty}(U)$, the space of smooth solenoidal vector fields with compact support in $U$. Since $a$ is dilation invariant, we apply this theorem to conclude that there exists a constant $C$ independent of $k$ such that

$$
\left|\nabla u_{k}\right| \leq C \quad \text { in } \quad U_{k} \times\left(-\left(t_{k}+1\right) M_{k}^{2}+1,0\right] .
$$

Note that since our $u$ vanishes on the boundary $u_{k}(\cdot, t) \in C_{0, \sigma}$ for all $k$ so Theorem 2.1 applies solutions starting from any $u_{k}(\cdot, t)$. We then conclude that $\left(u_{k}, \omega_{k}\right) \rightarrow(\bar{u}, \bar{\omega})$ as $k \rightarrow \infty$ *-weakly in $L^{\infty}$ with some $(\bar{u}, \bar{\omega})$ such that $|\bar{u}| \leq 1,|\bar{\omega}| \leq C$ in $(-\infty, 0] \times \mathbf{R}_{+,-c}^{3}$, up to rotation by taking a subsequence, where

$$
\mathbf{R}_{+,-c}^{3}=\left\{\left(x_{1}, x_{2}, x_{3}\right) \in \mathbf{R}^{3} \mid x_{3}>-c\right\} ;
$$

see e.g. [5] and [12, Proof of Theorem 6.2, Case 2] for the convergence to $\mathbf{R}_{+,-c}^{3}$. Here $c=\lim _{k \rightarrow \infty} c_{k}, c_{k}=M_{k} \operatorname{dist}\left(x_{k}, \partial U\right)$. In case $c=\infty$, we understand that $\mathbf{R}_{+,-c}^{3}=\mathbf{R}^{3}$. In both cases $\bar{u}$ is a bounded global mild solution of the Navier-Stokes equations in a half space with the zero Dirichlet boundary condition, if $c<\infty$ and in $\mathbf{R}^{3}$ if $c=\infty$. (This solution is often called an ancient solution.)

2.3. Local strong compactness. We would like to prove that $u_{k}$ converges to $\bar{u}$ locally uniformly near $(0,0)$ to conclude that $u_{k}(0,0) \rightarrow \bar{u}(0,0)$. Different from the whole space problem global higher derivative estimates are unavailable. We shall localize the problem. As pointed out in [14], it is 
convenient to estimate pressures to localize the problem. A key idea is to decompose pressure into two parts: a pressure coming from convective term called the convective pressure and a pressure coming from the viscosity term called the Stokes pressure as in J.-G. Lin-J. Lin-R. Pego [25]. By taking div of the momentum equation of (1.1), we see that $-\Delta p=\operatorname{div} \operatorname{div}(u \otimes u)$ since $\operatorname{div} u=0$. By taking inner products with the external unit normal $\mathbf{n}$ of $\partial U$ with the momentum equation we see that $\partial p / \partial \mathbf{n}=\mathbf{n} \cdot \Delta u$ on $\partial U$. Since $\operatorname{div} u=0$, one observes that $\mathbf{n} \cdot \Delta u=-\operatorname{div}_{\partial U}(\omega \times \mathbf{n})$, where $\operatorname{div} \partial \Omega$ denotes the surface divergence. Our pressure $p$ is decomposed as

$$
p=p_{F}+p_{S}
$$

satisfying

$$
\begin{array}{lllll}
-\Delta p_{F}=\operatorname{div} \operatorname{div}(u \otimes u) & \text { in } \quad U, & \partial p_{F} / \partial \mathbf{n}=0 \quad \text { on } \quad \partial U \\
-\Delta p_{S}=0 & \text { in } \quad U, & \partial p_{S} / \partial \mathbf{n}=-\operatorname{div}_{\partial U}(\omega \times \mathbf{n}) \quad \text { on } \quad \partial U .
\end{array}
$$

The pressure $p_{F}$ is called the convective pressure while $p_{S}$ is called the Stokes pressure. The next two lemmas on the Neumann problem or the Poisson and the Laplace equation is fundamental.

Lemma 2.3. Let $\Omega$ be a bounded $C^{2}$ domain in $\mathbf{R}^{n}$. Let $Z$ be the space of tensor fields on $\Omega$ of the form

$$
Z=\left\{f=\left(f_{i j}\right)_{1 \leq 1, j \leq n} \in C^{2}(\bar{\Omega}) \mid f=0 \text { on } \partial \Omega, \operatorname{div} f=0 \text { on } \partial \Omega\right\}
$$

Then there is a linear operator $N$ from $Z$ to $B M O\left(\mathbf{R}^{n}\right)$ such that $q=N f$ solves

$$
-\Delta q=\operatorname{div} \operatorname{div} f \text { in } \Omega, \quad \partial q / \partial \mathbf{n}=0 \text { on } \partial \Omega
$$

and that there exists a constant $C$ such that

$$
[q]_{B M O} \leq C\|f\|_{\infty} .
$$

Here $[q]_{B M O}$ denotes the $B M O$ semi-norm defined by

$$
[q]_{B M O}=\sup \left\{\frac{1}{\left|B_{r}(x)\right|} \int_{B_{r}(x)}\left|f(y)-f_{B_{r}(x)}\right| d y \mid x \in \mathbf{R}^{n}, r>0\right\},
$$

where $\left|B_{r}(x)\right|$ denotes the volume of the closed ball $B_{r}(x)$ with radius $r$ centered at $x$ and

$$
f_{B_{r}(x)}=\frac{1}{\left|B_{r}(x)\right|} \int_{B_{r}(x)} f(y) d y
$$

which is the average of $f$ over $B_{r}(x)$. We shall give its proof in the Appendix.

Lemma 2.4. Let $\Omega$ be a bounded $C^{2}$ domain in $\mathbf{R}^{n}$. Then there is a constant $C$ such that

$$
\left\|d_{\Omega} \nabla q\right\|_{\infty} \leq C\|g\|_{L^{\infty}(\partial \Omega)}
$$

holds for all $q \in C^{1}(\Omega)$ satisfying

$$
\Delta q=0 \text { in } \Omega, \quad \partial q / \partial n=\operatorname{div}_{\partial \Omega} g \text { on } \partial \Omega
$$


in a very weak sense where $g \in L^{\infty}(\partial \Omega)$ is tangential, i.e., $g \cdot \mathbf{n}=0$ on $\partial U$. Here $d_{U}(x)=\operatorname{dist}(x, \partial U)$.

This lemma is first proved in [5] for very weak solutions; in [5], the boundary regularity is assumed to be $C^{3}$ but can be weakened to $C^{2}[6]$. The same estimate has been established independently by Kenig-Lin-Shen [20] with a different expression.

We now continue to estimate rescaled pressure $p_{k}$ of the form

$$
p_{k}(x, t)=\lambda_{k}^{2} p\left(x_{k}+\lambda_{k} x, t_{k}+\lambda_{k}^{2} t\right) .
$$

We decompose $p_{k}=p_{F k}+p_{S k}$ and apply Lemma 2.3 and Lemma 2.4 to get

$$
\begin{aligned}
{\left[\tilde{p}_{F k}\right]_{B M O} } & \leq C_{1}\left\|u_{k}\right\|_{\infty}^{2} \\
\left\|d_{U_{k}} \nabla p_{S k}\right\|_{\infty} & \leq C_{2}\left\|\omega_{k}\right\|_{\infty},
\end{aligned}
$$

where $\tilde{p}_{F k}$ is the extension obtained by $N\left[u_{k} \otimes u_{k}\right]$. Since $U_{k}$ is obtained by translation and dilation of $U$, one can take constants $C_{1}, C_{2}$ independent of $k$. These estimates yield that

$$
\left\|p_{k}\right\|_{L^{s}\left(B_{R}\left(x_{0}\right) \cap U_{k}\right)} \leq C_{1}\left\|u_{k}\right\|_{\infty}^{2}+C_{2}\left\|\omega_{k}\right\|_{\infty} \leq C_{1}+C_{2} C
$$

since $\left\|u_{k}\right\|_{\infty} \leq 1,\left\|\omega_{k}\right\|_{\infty} \leq C$, if one normalizes $p_{k}$ such that

$$
\int_{B_{R}\left(x_{0}\right)} p_{k} d x=0
$$

where $x_{0} \in \mathbf{R}^{n}, s \in(1, \infty)$ with fixed $R$. As indicated in [14], the estimate (2.1) enables us to localize the problem and apply the $L^{s}$-maximum regularity results [16] of the Stokes problem in a domain $U_{k} \cap B_{R}\left(x_{0}\right)$. One has to mollify the corner so that $C^{2}$ regularity can be controlled uniformly in $k$. We conclude that $\left\{u_{k}\right\}$ is bounded in an anisotropic $L^{s}$-Sobolev space $W_{s}^{2,1}\left(\left(B_{R / 2}\left(x_{0}\right) \cap U\right) \times I\right)$ for all $s \geq 1$ when $I$ is a bounded time interval in $(-\infty, 0]$. By the Sobolev embedding [22, II Lemma 3.3], we know that the space-time Hölder norm of $\nabla u_{k}$ is bounded which implies that $\left(u_{k}, \omega_{k}\right)$ converges to $(\bar{u}, \bar{\omega})$ locally uniformly near $x_{0} \in \mathbf{R}^{n}$. Since $\left(x_{k}, t_{k}\right)$ is taken so that $\left|u_{k}(0,0)\right| \geq 1 / 2$, by the choice 2.1 (iii) we now conclude that $|\bar{u}(0,0)| \geq 1 / 2$. As noted in [14], $W_{s}^{2,1}$-bound implies regularity of pressure and a boot-strap argument implies uniform convergence of higher derivatives of $u_{k}$ but we do not use this property here.

2.4. Completion of the proof of Theorem 1.3. Since $\omega_{k}$ converges locally uniformly to $\bar{\omega}$, we now apply the continuous alignment condition at the place where $\bar{\omega} \neq 0$ to conclude that $\bar{\omega}$ pointed out just one fixed direction. This implies that the limit flow is two-dimensional with fixed sign of scalar vorticity; see [14], [15]. If $c=\infty$, the limit $\bar{u}$ is a mild solution of $\mathbf{R}^{2}$ and we apply the Liouville theorem as in [15] to get $\bar{u} \equiv 0$ which contradicts $|\bar{u}(0,0)| \geq 1 / 2$. If $c<\infty$, we apply the following Liouville theorem with boundary to get the same contradiction. The proof of Theorem 1.3 is now complete. 
Theorem 2.5 ([14], Theorem 1.1 and Remark 1.2). Let $(u, p)$ be a bounded mild solution to the Navier-Stokes system in $\mathbf{R}_{+}^{2} \times(-\infty, 0)$. Assume that $\sup _{-\infty<t<0}(-t)^{1 / 2}\|u(t)\|_{\infty}<\infty$ and $\omega \geq 0$ in $\mathbf{R}_{+}^{2} \times(-\infty, 0)$. Then $u$ is identically equal to zero.

We note that because of the pressure estimates the limit $(\bar{u}, \bar{p})$ is not only a solution of the Navier-Stokes equations but also the pressure has a special structure which guarantees that $(\bar{u}, \bar{p})$ is a mild solution. We are able to apply this theorem to get a contradiction also for $c<\infty$.

\section{Appendix}

We shall prove Lemma 2.3. We first recall the Green function of the Neumann problem, which is often called the Neumann function denoted by $N=N(x, y), x, y \in \bar{\Omega}$. It is defined by a unique solution of

$$
\begin{aligned}
-\Delta_{x} N(x, y) & =\delta(x-y)-|\Omega|^{-1} \quad \text { in } \quad \Omega \\
\frac{\partial N}{\partial \mathbf{n}_{x}}(x, y) & =0 \quad \text { on } \quad \partial \Omega, \quad \int_{\Omega} N(x, y) d x=0, \quad y \in \Omega,
\end{aligned}
$$

where $\Omega$ is a domain in $\mathbf{R}^{n}$. Let $E$ denote the fundamental solution of $-\Delta$, i.e.,

$$
\begin{aligned}
& E(x)=-\frac{1}{2 \pi} \log |x| \text { for } n=2, \\
& E(x)=-\frac{1}{(n-2)\left|S^{n-1}\right||x|^{n-2}} \quad \text { for } n \geq 3 .
\end{aligned}
$$

Lemma 3.1. Let $\Omega$ be a bounded $C^{2}$ domain in $\mathbf{R}^{n}$. Then the Neumann function $N$ is of the form:

$$
N(x, y)=E(x-y)+h(x, y)
$$

with $h \in C^{\infty}(\Omega \times \Omega)$ satisfying $h(x, y)=h(y, x)$ and

$$
\sup _{x \in \Omega} \int_{\Omega}\left|\nabla_{y}^{k} h(x, y)\right|^{1+\delta} d y<\infty \quad \text { for } \quad k=0,1,2 .
$$

for sufficiently small $\delta>0$ depending only on $n$.

We postpone the proof at the end of Appendix.

Proof of Lemma 2.3. For $f \in Z$ we set

$$
\left(N_{0} f\right)(x)=\int_{\Omega} N(x, y) \operatorname{div} \operatorname{div} f(y) d y, \quad x \in \Omega .
$$

Integrate by parts to get

$$
\left(N_{0} f\right)(x)=\sum_{i, j} \text { p.v. } \int_{\Omega} \partial_{y_{i}} \partial_{y_{j}} N(x, y) f_{i j}(y) d y
$$


since $f=0$ and $\operatorname{div} f=0$ on $\partial \Omega$. Here we take the principal value p.v. since $\partial_{i} \partial_{j} E$ is a singular integral

$$
\begin{aligned}
\left(N_{0} f\right)(x) & =\sum_{i, j} \text { p.v. } \int_{\Omega} \partial_{x_{i}} \partial_{x_{j}} E(x-y) f_{i j}(y) d y \\
& +\sum_{i, j} \int_{\Omega} \partial_{y_{i}} \partial_{y_{j}} h(x, y) f_{i j}(y) d y \equiv N_{0 S} f+N_{0 R} f
\end{aligned}
$$

by symmetry of $N$, i.e., $N(x, y)=N(y, x)$. We extend a singular part $N_{0 S} f$ and regular part $N_{0 R} f$ to outside $\Omega$ in a way such that

$$
\begin{aligned}
& \left(N_{S} f\right)(x)=\sum_{i, j} \text { p.v. } \int_{\Omega} \partial_{x_{i}} \partial_{x_{j}} E(x-y) f_{i j}(y) d y, \quad x \in \mathbf{R}^{n} \\
& \left(N_{R} f\right)(x)=0, \quad x \in \Omega^{c} \quad \text { and } \quad\left(N_{R} f\right)(x)=\left(N_{0 R} f\right)(x), \quad x \in \Omega .
\end{aligned}
$$

Evidently, $N f=N_{0} f$ in $\Omega$. By $B M O-L^{\infty}$ estimate for the singular integral operator [17], we see that

$$
\left[N_{S} f\right]_{B M O} \leq C\|f\|_{\infty} .
$$

By Lemma 3.1, we see that

$$
\begin{aligned}
\left\|N_{R} f\right\|_{\infty} & \leq \sup _{x \in \Omega} \int_{\Omega}\left|\nabla_{y}^{2} h(x, y)\right| d y\|f\|_{\infty} \\
& \leq C^{\prime}\|f\|_{\infty}
\end{aligned}
$$

since $\Omega$ is bounded. By definition of the Neumann function, this $N f$ is the desired solution of the Neumann problem. Thus the proof is now complete.

Proof of Lemma 3.1. If $N(x, y)$ is given in the form of Lemma 3.1, then $h$ must solve

$$
\Delta_{x} h=|\Omega|^{-1} \quad \text { in } \quad \Omega, \quad \partial h / \partial \mathbf{n}_{x}=-\partial E(x, y) / \partial \mathbf{n}_{x}=: g^{y} \quad \text { in } \quad \partial \Omega .
$$

Moreover, $h$ satisfies

$$
\int_{\Omega} h(x, y) d x=-\int_{\Omega} E(x-y) d x=: c_{y} .
$$

Since $\Omega$ is bounded, $\left|c_{y}\right|$ is bounded in $\Omega$. We notice that there exists a constant such that

$$
\left|g^{y}(x)\right|=\left|\mathbf{n}(x) \cdot \nabla_{x} E(x-y)\right| \leq \frac{C}{|x-y|^{n-2}}
$$

for $x \in \Gamma=\partial \Omega$ near $y \in \Gamma$ since $\Gamma$ is $C^{2}$; see e.g. [18, p.140]. Note that the singularity near $y \in \Gamma$ is milder than $|x-y|^{1-n}$. Since $\Omega$ is bounded, we are able to take such a constant $C$ independent of $x \in \Gamma$ and $y \in \bar{\Omega}$. A similar estimate is available for the tangential derivative of $g^{y}$. In fact, there is a constant $C$ such that

$$
\left|\nabla_{\Gamma} g^{y}\right| \leq \frac{C}{|x-y|^{n-1}}
$$


for any $x \in \Gamma, y \in \bar{\Omega}$. Since a function $f \in C_{c}^{1}\left(\mathbf{R}^{d} \backslash\{0\}\right)$ satisfying

$$
|f| \leq \frac{C}{|x|^{d-1}}, \quad|\nabla f| \leq \frac{C}{|x|^{d}}
$$

belongs to the $L^{1}$ Sobolev space of order $1-\eta$, i.e., $f \in W^{1-\eta, 1}\left(\mathbf{R}^{d}\right)$ for any $\eta \in(0,1)$ (see Lemma 3.2 below), we see that $g^{y} \in W^{1-\eta, 1}(\Gamma)$ and $\left\|g^{y}\right\|_{W^{1-\eta, 1}}$ is bounded independent of $y \in \bar{\Omega}$ by taking $d=n-1$. Since $W^{1-\eta, 1}$ equals the Besov space $B_{1,1}^{1-\eta}$, by the Sobolev embedding of Besov spaces (see e.g. [28]), we see that $g^{y} \in W^{1-1 / p, p}(\partial \Omega)$ for $p>1$ sufficiently close to one.

By applying the standard $L^{p}$ estimate for the Neumann problem, we see that there is a constant $C_{0}$ depending only on $\Omega$ and $p$ such that

$$
\left\|h(\cdot, y)-c_{y}\right\|_{W^{2, p}\left(\Omega_{x}\right)} \leq C_{0}\left(\left\|g^{y}\right\|_{W^{1-1 / p, p}\left(\partial \Omega_{x}\right)}+\|\varphi\|_{L^{p}(\Omega)}\right) .
$$

with a constant function $\varphi=|\Omega|^{-1}$. This follows from an a priori estimate (by setting $h_{0}=h(\cdot, y)-c_{y}$ ) for the solution $h_{0}$ of the Neumann problem

$$
-\Delta h_{0}=f \quad \text { in } \quad \Omega, \quad \partial h_{0} / \partial \mathbf{n}=g, \quad \int_{\Omega} h_{0} d x=0
$$

in a bounded smooth domain $\Omega$ of the form

$$
\left\|h_{0}\right\|_{W^{2, p}(\Omega)} \leq C_{1}\left(\|f\|_{L^{p}(\Omega)}+\|g\|_{W^{1-1 / p, p}(\Gamma)}\right) .
$$

Indeed, this follows from general a priori estimate

$$
\left\|h_{0}\right\|_{W^{2, p}(\Omega)} \leq C_{2}\left(\|f\|_{L^{p}(\Omega)}+\|g\|_{W^{1-1 / p, p}(\Gamma)}+\|h\|_{L^{p}(\Omega)}\right),
$$

found in [1]; see also [31, Theorem 4.10]. Here the constant $C_{j}(j=1,2)$ depends only on $\Omega$ and $p$. Although the argument is standard, we give a proof of deriving (3.2) from (3.3) for completeness. Suppose (3.2) were false, then there would exist a sequence $\left\{h_{m}, g_{m}, f_{m}\right\}$ such that

$$
1=\left\|h_{m}\right\|_{W^{2, p}}>m\left(\left\|f_{m}\right\|_{L^{p}}+\left\|g_{m}\right\|_{W^{1-1 / p, p}}\right) .
$$

This implies that $\left\|f_{m}\right\|_{L^{p}},\left\|g_{m}\right\|_{W^{1-1 / p, p}}$ tends to zero. By Rellich's compactness theorem, we see that $h_{m} \rightarrow h$ in $L^{p}$ strongly with some $h \in W^{2, p}(\Omega)$ by taking a subsequence. The a priori estimate (3.3) now implies that $\|h\|_{p} \geq 1 / C_{2}$. However, since $h$ is a unique solution of the homogeneous Neumann problem if one imposes the average zero condition, $h$ must be zero. This is a contradiction. We now obtain (3.2).

Since $\Omega$ is bounded, the right-hand side of (3.1) is bounded uniformly in $y$. Since $\left|c_{y}\right|$ is bounded, we see

$$
\sup _{y \in \Omega} \int\left|\nabla_{x}^{k} h(x, y)\right|^{p} d x, \quad k=0,1,2
$$

is finite. Since $h$ is symmetric, the desired estimate holds by changing $x$ and $y$. 
Lemma 3.2. Assume that $f \in C^{1}\left(\mathbf{R}^{d} \backslash\{0\}\right)(d \geq 1)$ satisfies

$$
|f(x)||x|^{d-1} \leq c_{1}, \quad|\nabla f(x)||x|^{d} \leq c_{2}
$$

with some constants $c_{1}$ and $c_{2}$ independent of $x \in \mathbf{R}^{d}$. Then for $s \in(0,1)$, $R>0$ the estimate

$$
I=\iint_{|x|,|y| \leq R} \frac{|f(x)-f(y)|}{|x-y|^{d+s}} d x d y \leq M<\infty
$$

holds with a constant $M$ depending only on $d, s, c_{1}, c_{2}$ and $R$. In particular,

$$
f \in W^{s, 1}\left(B_{R}(0)\right) .
$$

Proof. Assume that $|x| \leq|y|$ and connect $x$ and $y$ by a geodesic curve in $B_{|x|}(0)^{c}$. Since the curve length is less than $(\pi / 2)|x-y|$, by a fundamental theorem of calculus, we observe that

$$
\begin{aligned}
|f(x)-f(0)| & \leq(\pi / 2)|x-y| \sup \left\{|\nabla f(z)| \mid z \in B_{|x|}(0)^{c}\right\} \\
& \leq(\pi / 2) c_{2}|x-y||x|^{-d} .
\end{aligned}
$$

Since the integrand of $I$ is symmetric with respect to $x$ and $y$, we now estimate

$$
\frac{I}{2}=\iint_{D_{1}}+\iint_{D_{2}} \frac{|f(x)-f(y)|}{|x-y|^{d+s}} d x d y=I_{1}+I_{2}
$$

with

$$
\begin{aligned}
& D_{1}=\{(x, y)|| x|\leq| y|\leq R,| x-y|\leq| x \mid\}, \\
& D_{2}=\{(x, y)|| x|\leq| y|\leq R,| x-y|\geq| x \mid\} .
\end{aligned}
$$

To estimate $I_{1}$, we observe that

$$
\begin{aligned}
\frac{|f(x)-f(y)|}{|x-y|^{d+s}} & \leq(\pi / 2) c_{2}|x|^{-d}|x-y|^{-d+1-s} \\
& \leq(\pi / 2) c_{2}|x|^{-d+\delta}|x-y|^{-d+1-\delta-s}
\end{aligned}
$$

for $\delta>0$ since $|x-y| \leq|x|$. We take $\delta$ such that $\delta<1-s$ so that

$$
\iint_{|x|,|y| \leq R}|x|^{-d+\delta}|x-y|^{-d+1-\delta-s} d x d y<\infty .
$$

since $d-\delta<d, d-1+\delta+s<d$. We thus observe that

$$
I_{1} \leq K c_{2}
$$

with $K$ depending only on $d, s, \delta$ and $R$.

To estimate $I_{2}$, we observe that

$$
\frac{|f(x)-f(y)|}{|x-y|^{d+s}} \leq \frac{|f(x)|+|f(y)|}{|x-y|^{d+s}} \leq 2 c_{1}|x-y|^{-d-s}|x|^{-d+1}
$$

since $|x| \leq|y|$. Since $|x-y| \geq|x|$, we take $\delta \in(s, 1)$ and observe that

$$
|x-y|^{-d-s}|x|^{-d+1} \leq|x-y|^{-d-s+\delta}|x|^{-d+1-\delta} .
$$


The last function is integrable on $B_{R}(0) \times B_{R}(0)$. Thus

$$
I_{2} \leq K^{\prime} c_{1}
$$

with $K^{\prime}$ depending only on $d, s, \delta$ and $R$. We thus conclude that

$$
I \leq 2\left(K^{\prime} c_{1}+K c_{2}\right) \text {. }
$$

\section{REFERENCES}

[1] S. Agmon, A. Douglis and L. Nirenberg, Estimates near the boundary for solutions of elliptic partial differential equations satisfying general boundary conditions. I. Comm. Pure Appl. Math. 12 (1959), 623-727.

[2] K. Abe, The Navier-Stokes equations in a space of bounded functions. Comm. Math. Phys. 338 (2015), 849-865.

[3] K. Abe, On estimates for the Stokes flow in a space of bounded functions. J. Differential Equations 261 (2016), 1756-1795.

[4] K. Abe, Exterior Navier-Stokes flows for bounded data. Math. Nachr. 290 (2017), 972-985.

[5] K. Abe and Y. Giga, Analyticity of the Stokes semigroup in spaces of bounded functions. Acta Math. 211 (2013), 1-46.

[6] K. Abe and Y. Giga, The $L^{\infty}$-Stokes semigroup in exterior domains. J. Evol. Equ. 14 (2014), 1-28.

[7] H.-O. Bae and B. J. Jin, Existence of strong mild solution of the Navier-Stokes equations in the half space with nondecaying initial data. J. Korean Math. Soc. 49 (2012), 113-138.

[8] H. Beirão da Veiga, Vorticity and regularity for flows under the Navier boundary condition. Commun. Pure Appl. Anal. 5 (2006), 907-918.

[9] H. Beirão da Veiga, Vorticity and regularity for viscous incompressible flows under the Dirichlet boundary condition. Results and related open problems. J. Math. Fluid Mech. 9 (2007), 506-516.

[10] H. Beirão da Veiga and L. C. Berselli, On the regularizing effect of the vorticity direction in incompressible viscous flows. Differential Integral Equations 15 (2002), 345-356.

[11] H. Beirão da Veiga, Y. Giga and Z. Grujić, Vorticity directions and regularity of solutions to the Navier-Stokes equations. Handbook of Mathematical Analysis in Mechanics of Viscous Fluids (eds. Y. Giga and A. Novotny), Springer (2018), 901931.

[12] M. Bolkart and and Y. Giga, On $L^{\infty}-B M O$ estimates for derivatives of the Stokes semigroup. Math. Z. 284 (2016), 1163-1183.

[13] P. Constantin and C. Fefferman, Direction of vorticity and the problem of global regularity for the Navier-Stokes equations. Indiana Univ. Math. J. 42 (1993), 775789 .

[14] Y. Giga, P.-Y. Hsu and Y. Maekawa, A Liouville theorem for the planer Navier-Stokes equations with the no-slip boundary condition and its application to a geometric regularity criterion. Comm. in Partial Differential Equations 39 (2014), 1906-1935.

[15] Y. Giga and H. Miura, On vorticity directions near singularities for the Navier-Stokes flows with infinite energy. Comm. Math. Phys. 303 (2011), 289-300.

[16] Y. Giga and H. Sohr, Abstract $L^{p}$ estimates for the Cauchy problem with applications to the Navier-Stokes equations in exterior domains. J. Functional Anal. 102 (1991), $72-94$.

[17] L. Grafakos, Modern Fourier analysis. Third edition. Graduate Texts in Mathematics, 250. Springer, New York, 2014. xvi+624 pp. 
[18] Q. Han and F. Lin, Elliptic partial differential equations. Second edition. Courant Lecture Notes in Mathematics, 1. Courant Institute of Mathematical Sciences, New York; American Mathematical Society, Providence, RI, 2011. x+147 pp.

[19] P.-Y. Hsu, H. Notsu and T. Yoneda, A local analysis of the axisymmetric NavierStokes flow near a saddle point and no-slip flat boundary. J. Fluid Mech. 794 (2016), 444-459.

[20] C. E. Kenig, F. Lin and Z. Shen, Homogenization of elliptic systems with Neumann boundary conditions. J. Amer. Math. Soc. 26 (2013), 901-937.

[21] G. Koch, N. Nadirashvili, G. Seregin and V. Šverák, Liouville theorems for the NavierStokes equations and applications. Acta Math. 203 (2009), 83-105.

[22] O. A. Ladyženskaja, V. A. Solonnikov and N. N. Ural'ceva, Linear and quasilinear equations of parabolic type. Vol. 23 American Mathematical Society, Providence, R.I. 1968. xi+648 pp.

[23] Z. Lei and Q. S. Zhang, A Liouville theorem for the axially-symmetric Navier-Stokes equations. J. Funct. Anal. 261 (2011), 2323-2345.

[24] Z. Lei, Q. S. Zhang and N. Zhao, Improved Liouville theorems for axially symmetric Navier-Stokes equations. Science China Mathematics 47 (2017), 1-16.

[25] J.-G. Liu, J. Liu and R. L. Pego, Stability and convergence of efficient Navier-Stokes solvers via a commutator estimate. Comm. Pure Appl. Math. 60 (2007), 1443-1487.

[26] P. Maremonti, Stokes and Navier-Stokes problems in the half-space: existence and uniqueness of solutions non converging to a limit at infinity. Zap. Nauchn. Sem. S.Peterburg. Otdel. Mat. Inst. Steklov. (POMI) 362 (2008), 176-240; translation in J. Math. Sci. (N.Y.) 159 (2009), 486-523.

[27] P. Maremonti, Non-decaying solutions to the Navier Stokes equations in exterior domains: from the weight function method to the well posedness in $L^{\infty}$ and in Hölder continuous functional spaces. Acta Appl. Math. 132 (2014), 411-426.

[28] Y. Sawano, Theory of Besov spaces. Developments in Mathematics, 56. Springer, Singapore, 2018. xxiii +945 pp.

[29] G. Seregin and V. Šverák, Regularity criteria for Navier-Stokes solutions. Handbook of Mathematical Analysis in Mechanics of Viscous Fluids (eds. Y. Giga and A. Novotny), Springer (2018), 829-866.

[30] V. A. Solonnikov, On nonstationary Stokes problem and Navier-Stokes problem in a half-space with initial data nondecreasing at infinity. J. Math. Sci. 114 (2003), $1726-1740$.

[31] H. Tanabe, Functional analytic methods for partial differential equations. Monographs and Textbooks in Pure and Applied Mathematics, 204. Marcel Dekker, Inc., New York, 1997. $\mathrm{x}+414$ pp.

Graduate School of Mathematical Sciences, The University of Tokyo, 3-

8-1 Komaba Meguro-Ku TOKyo 153-8914, Japan

E-mail address, Y. Giga: labgiga@ms.u-tokyo.ac.jp

E-mail address, Z. Gu: guzy@ms.u-tokyo.ac.jp

E-mail address, P.-Y. Hsu: pyhsu@ms.u-tokyo.ac.jp 